\title{
Simulation of Dynamic Reactive Power Compensation Device Based on Variable Reactance Converter
}

\author{
Mengjie TU ${ }^{a}$, Youxin YUAN ${ }^{b}$, Jing $C H E N^{c}$, Ying $\operatorname{TAN}^{d}$ \\ School of Automation, Wuhan University of Technology, Wuhan, 430070, China \\ aemail:tmjtutu@163.com, bemail:yyx2000@263.net, cemail:jingchen680@163.com, demail:5640829 \\ 93@qq.com
}

Keywords: Dynamic Reactive Power Compensation; Variable Reactance Converter; Simulation

\begin{abstract}
In the power system, the reactive power causes low power factor and power loss. The reactive power compensation device can ensure normal and stable operation of the power system. The traditional reactive power compensation device is difficult to meet the needs of high power factor and low power loss. Therefore, a dynamic reactive power compensation device has been presented by authors to achieve high power factor and low power loss. Based on the previous research results, the dynamic reactive power compensation device based on variable reactance converter is simulated. The following works have been done in the study: topology of dynamic reactive power compensation device, simulation model of dynamic reactive power compensation device, and simulation. The simulation results show that the dynamic reactive power compensation device based on variable reactance converter improves the power factor of power system and saves energy. The research of this paper has laid a theoretical foundation for the dynamic reactive power compensation device in practical applications.
\end{abstract}

\section{Introduction}

There are many inductive loads in the power system. They absorb the reactive power. It causes power loss and low power factor. The reactive power compensation device can improve the power factor of power system and reduce the power loss. At present, the reactive power compensation device includes the synchronous compensatory, the saturable reactor, the thyristor controlled reactor, the thyristor switched capacitor and the static var compensator [1].

The dynamic reactive power compensation device doesn't adopt the structure of series anti-parallel thyristors and reactor. It consists of variable reactance converter and capacitor banks. The reactive power is compensated by switching capacitor banks and adjusting variable reactance converter dynamically. The compensation mode is dynamic and smooth [2].

Based on the previous research results, the dynamic reactive power compensation device based on variable reactance converter is simulated. The following works have been done in the study: topology of dynamic reactive power compensation device, simulation model of dynamic reactive power compensation device, and simulation.

\section{Topology of Dynamic Reactive Power Compensation Device}

The topology of the dynamic reactive power compensation device is shown in Fig.1. In Fig.1, the dynamic reactive power compensation device consists of the capacitor banks $\left(C_{B}\right)$, the variable reactance converter $\left(L_{V}\right)$ and the intelligent controller (ICC) [3]. The load $\left(F_{Z}\right)$ is connected to the primary side of $L_{V}$, and the secondary side is connected to the power converter (PCC).

The required compensation capacity $Q_{B}$ of the power system is expressed as follow:

$$
Q_{B}=P\left(\sqrt{\frac{1}{\cos ^{2} \varphi_{1}}-1}-\sqrt{\frac{1}{\cos ^{2} \varphi_{2}}-1}\right)
$$

Where, $P$ is the rated active power, $\cos \varphi_{1}$ is the power factor of $F_{Z}$, and $\cos \varphi_{2}$ is the target power factor. 


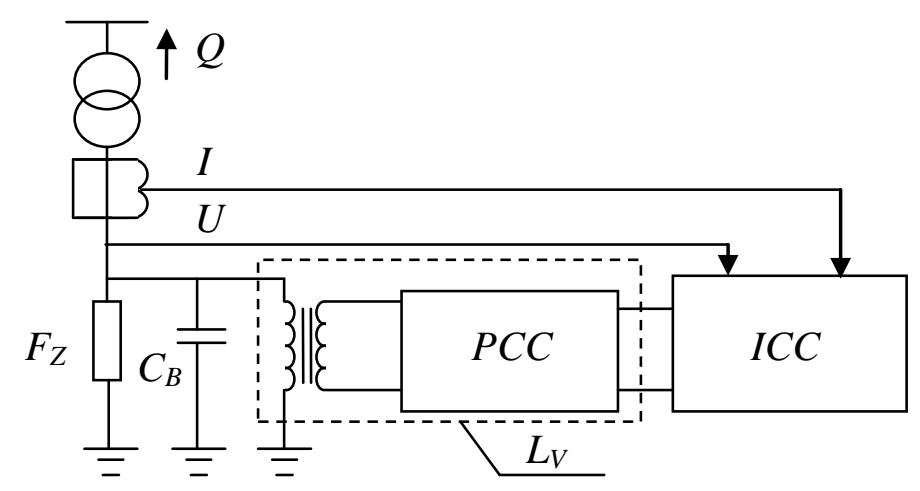

Fig.1. Topology of dynamic reactive power compensation device

witched on appropriately to produce reactive power $Q_{C}$ based on $Q_{B}$. It can increase the power factor close to $\cos \varphi_{2}$. In practical case, $C_{B}$ is difficult to meet the needs of $Q_{B}$. When it is over-compensated, $L_{V}$ is adjusted to absorb the amount of overcompensation reactive power $\left(Q_{L}\right)$ [4]. The inductance value of $L_{V}$ is adjusted continuously by adjusting the control signal of PCC. In this way, $C_{B}$ is cooperated with $L_{V}$ to realize the fast, dynamic and smooth reactive power compensation [5].

\section{Simulation of Dynamic Reactive Power Compensation Device}

According to the topology of dynamic reactive power compensation device, a simulation model is constructed by MATLAB/Simulink tool. The simulation model of dynamic reactive power compensation device is shown in Fig.2.

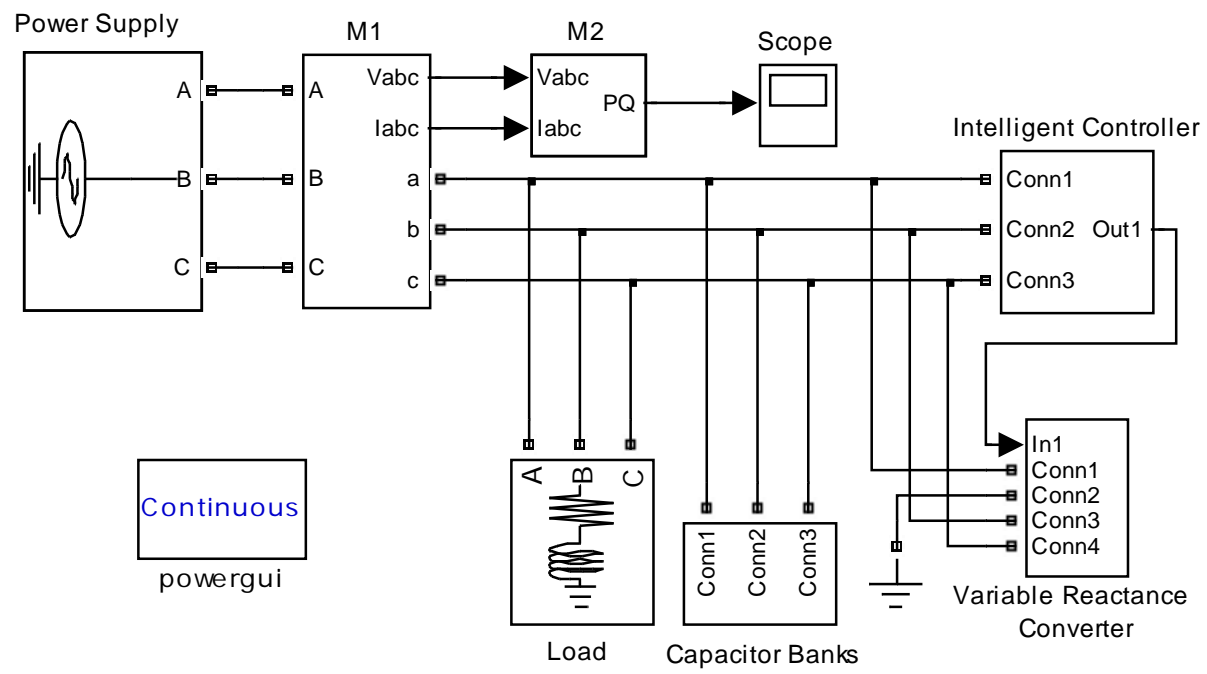

Fig.2. Simulation model of dynamic reactive power compensation device

As shown in Fig.2, the three-phase source is used in the power supply model. The phase voltage and phase current are detected by three-phase V-I measurement $\left(M_{1}\right)$. The active power and reactive power of system are detected by three-phase instantaneous active \& reactive power measurement $\left(M_{2}\right)$. The principle of variable reactance converter is similar to the transformer. Therefore, the variable reactance converter model consists of a three-phase transformer and the anti-parallel thyristors. The synchronized 6-pulse generator is used in the intelligent controller module.

Basing on the topology of dynamic reactive power compensation device, the power supply, the load and the capacitor banks are connected to the primary side of variable reactance converter. The intelligent controller is connected to the secondary side of variable reactance converter. 


\section{Analysis of Simulation Results}

The considered system parameters are described in the following: the phase-to-phase rms voltage is $380 \mathrm{~V}$, the frequency is $50 \mathrm{~Hz}$, the rated power of load is $100 \mathrm{~kW}$ and the simulation stop time is $0.08 \mathrm{~s}$.

Assume that the rated power of load is constant and the inductive reactive power $\left(Q_{L}\right)$ changes in a range of $0 \sim 100 \mathrm{kVar}$. The curve of the power factor is shown in Fig.3.

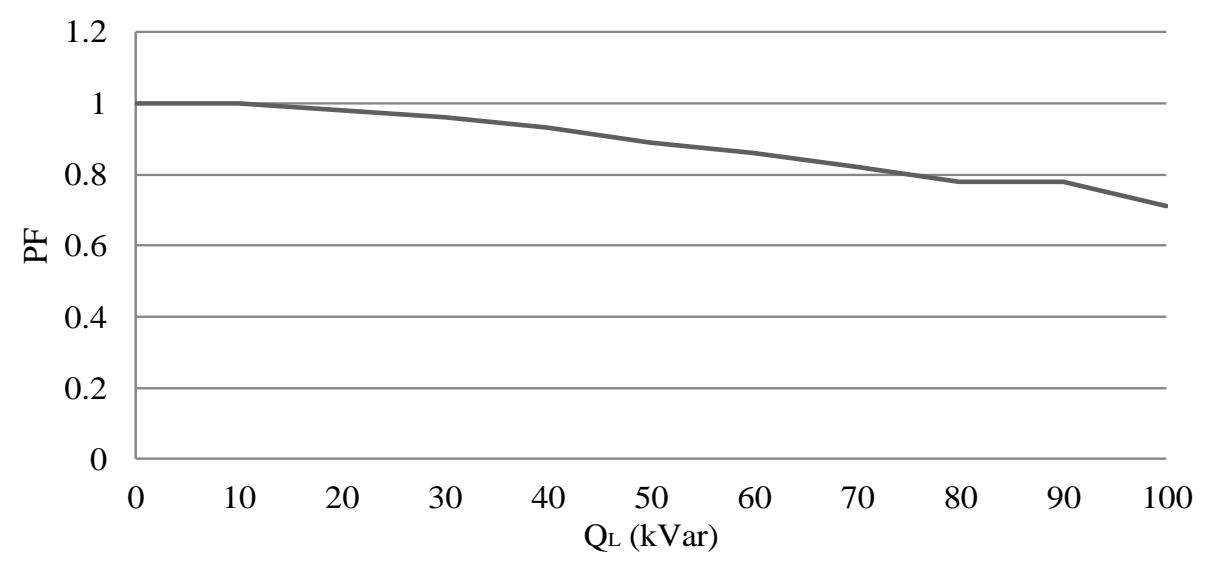

Fig.3. Curve of the power factor

As shown in Fig.3, the power factor of system decreases when the conductive reactive power of load increases. If the power factor is too low, the electrical equipment will run abnormally. It also influences the power quality and stability of the power system.

Assume that the power factor of $F_{Z}$ is 0.75 . The target power factor is 0.95 . According to Eq.1, $Q_{B}$ and $Q_{C}$ are respectively $55 \mathrm{kVar}$ and $80 \mathrm{kVar}$. At the same time, $L_{V}$ is adjusted to absorb the amount of overcompensation reactive power $Q_{L}$. The curves of the active and reactive power are respectively shown in Fig.4 and Fig.5.

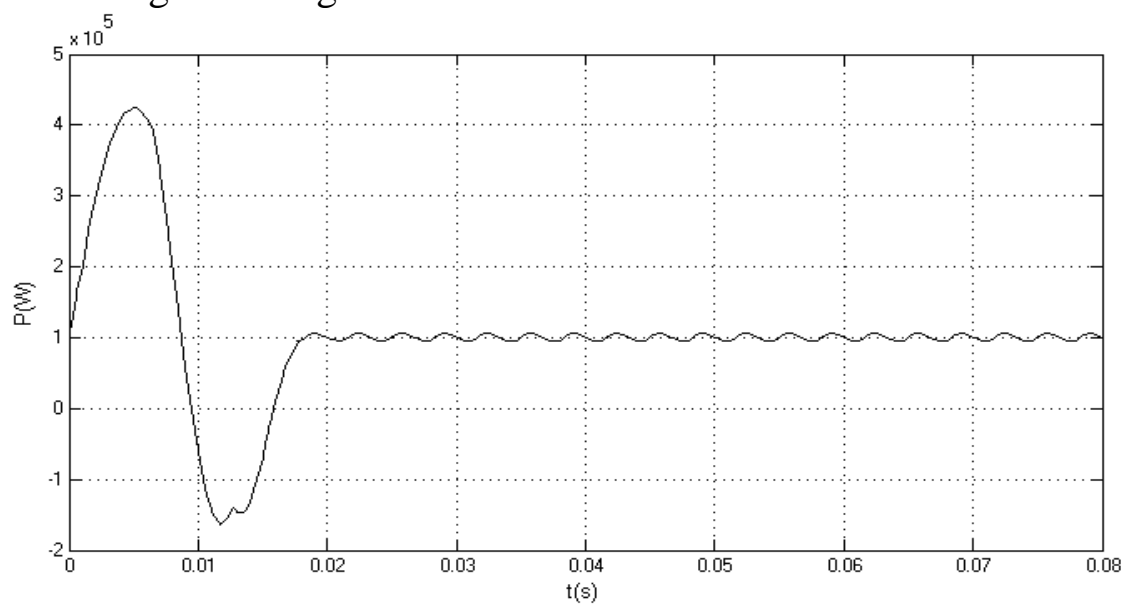

Fig.4. Curve of the active power 


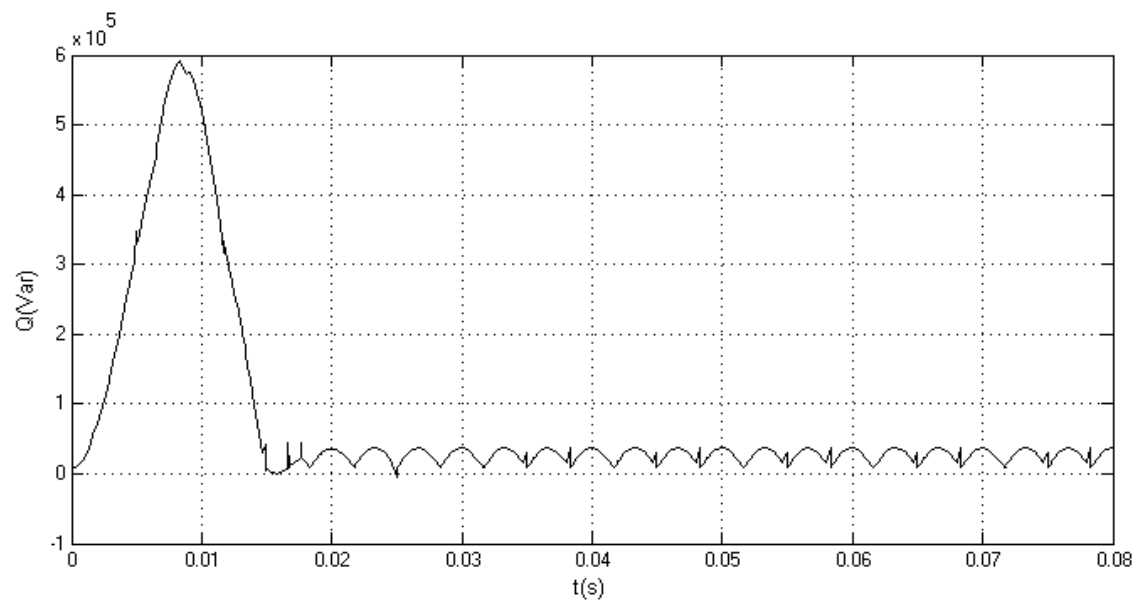

Fig.5. Curve of the reactive power

As can be seen from Fig.4 and Fig.5, the target power factor of the power system reaches 0.95 . The simulation curves show that dynamic reactive power compensation device based on variable reactance converter improves the power factor of power system. At the same time, it saves energy and stabilizes the voltage of the power system.

\section{Conclusions}

In the power system, the reactive power causes lower power factor and power loss. Therefore, a dynamic reactive power compensation device has been presented by authors to achieve high power factor and low power loss. Based on the previous research results, the dynamic reactive power compensation device based on variable reactance converter is simulated. The following works have been done in the study: topology of dynamic reactive power compensation device, simulation model of dynamic reactive power compensation device, and simulation. The simulation results show that the dynamic reactive power compensation device based on variable reactance converter improves the power factor of power system and saves energy. The research of this paper has laid a theoretical foundation for the dynamic reactive power compensation device in practical applications.

\section{Acknowledgements}

This work is financially supported by the projects of Hubei Science and Technology Plans of China (Granted No: 2013BAA009).

\section{References}

[1] Youxin Yuan, Shanshan Cai, Juquan Ban, Tanfu Xiao. Research on Topology of the Static Reactive Power Compensator Based on the Controlled Reactance [J]. Journal of Wuhan University of Technology, 200931 (12) 120-122.

[2] Youxin Yuan, Yiping Xiao. Research on Impedance Conversion Mechanism of Variable Reactor [J]. Journal of Wuhan University of Technology, 200830 (3) 133-135.

[3] Na Xie, Youxin Yuan, Xianglian Xu, Hongming Hu. Research on Static Var Compensator Mechanism of Compensation Based on Reactor Variable [J]. Journal of Wuhan University of Technology, 200932 (10) 129-132.

[4] Yufang Chang, Youxin Yuan, Yan Xu, Wencong Huang. Topological Structure and Impedance Conversion Analysis of Power Electronic Reactor [J]. Power Electronics, 201246 (2) 21-23.

[5] Yufang Chang, Youxin Yuan, Wencong Huang, and Yan Xu. Research on Impedance Conversion of Power Electronic Reactor [J]. Journal of Wuhan University of Technology, 201133 (10) $127-130$. 